are more strongly correlated to $\sigma_{f}$ than to each other. However, this will probably not be true for most choices of $f$ since for $f$ distant from $g$, the sites $l, m$ and $n$ must be much closer to each other (since they are clustered around $g$ ) than they are to $f$, and Cowley's approximation for this term should be considerably in error. On the other hand Cowley's theory does work quite well for most systems. The reason for this is probably the fact that at temperatures sufficiently above the ordering temperature, only the term linear in $\beta$ gives a significant contribution. This suggests that the expression derived from (8) by retaining only the first term:

$$
\alpha_{g}=-\frac{1}{2} \beta \sum_{h} V_{0 h} \alpha_{h}
$$

should be as good as Cowley's approximation and has the advantage of being much simpler to solve.

The expression of Christy and Hall is obtained from (8) by retaining only the correlations of $\sigma_{f}$ with itself and does not give even the first term in the exact high temperature expansion for $\left\langle\sigma_{f} \sigma_{g}\right\rangle$ correctly.

I am deeply indebted to Prof. Simon Moss for introducing me to the current theories of order in alloy systems and for many interesting discussions. Prof. Moss and the author are currently working on an analogous discussion for the case of binary alloys of general composition and its application to the experimental measurements of the short range order parameters in several alloy systems.

\title{
ATTAINMENT OF HIGH RESOLUTIONS IN WAVEFRONT-RECONSTRUCTION IMAGING
}

\author{
G. W. STROKE and D. G. FALCONER \\ The University of Michigan, Ann Arbor, Michigan
}

Received 24 November 1964

This letter gives a theoretical analysis and some experimental illustrations for the extension to the $\mathrm{X}$-ray domain of wavefront reconstruction imaging principles [1-6] *, for the cases where high resolutions, as well as high magnifications and faithful recording of the phases in the diffracted wavefronts are necessary. High magnifications, phase recordings, as well as high resolutions have all been previously demonstrated in X-ray work [7-9], but high resolutions did not heretofore appear attainable in comparable image-forming $X$-ray microscopy using heretofore described holographic wavefront reconstruction methods $[1-5,10]$. Indeed, resolutions of only $5000 \AA$ to $10000 \AA$, rather than $1 \AA$, appeared heretofore attainable by means of "conventional" wavefront reconstruction methods.

The basic theory of high-resolution micro-holography (wavefront reconstruction imaging) can be given by means of the following one-dimensional model. Let $T(\xi)$ be a small periodic structure whose complex amplitude transmittance is

$$
T(\xi)=\operatorname{rect}\left(\frac{\xi}{l}\right) \sum_{n} A_{n} \exp \left[\mathbf{i}\left(\varphi_{n}+n \beta \xi\right)\right]
$$

where $2 l(\beta)$ is the length (period) of the structure, and $A_{n} \exp \left(\mathrm{i} \varphi_{n}\right)$ are the constant Fourier coefficients **. When $T(\xi)$ is illuminated by a plane wave (wave-number $k$ ) it will act as a diffraction grating

* For a recent review of this field, see e.g. ref. 6.

** The rect function is defined in the usual way as a unit transmission function over the extent of the argument [11]. See also refs. 6 and 12). 
and produce a number of diffraction orders (one for each Fourier component) in an $x$-plane ( $x$ parallel to $\xi)$. If a coherent reference beam is superposed at an angle $\theta$ on the scattered field according to refs. 4 and 5 , the resultant amplitude $A(x)$ will be proportional to

$$
A_{0} \exp [-1 \alpha x]+\sum_{n} \text { rect }\left(\frac{x}{l}-\frac{f n \beta}{k l}\right) A_{n} \exp \left[\mathrm{i}\left(\varphi_{n}+n \beta x-\frac{f n^{2} \beta^{2}}{2 k}\right)\right] .
$$

where $k$ is the wave-number of the radiation, $A_{0}$ is a large constant representing the coher ent background radiation, and $\alpha=k \theta$. The $n$th order has been diffracted upwards along $x$ by a distance according to conventional grating theory [13]. The intensity $I(x)$ which would be incident on the $x$-axis under these conditions would be proportional to

$$
I(x) \propto A_{0}^{2}+\sum_{n} \text { rect }\left(\frac{x}{l}-\frac{f n \beta}{k l}\right) A_{n}^{2}-2 A_{0} \sum_{n} \text { rect }\left(\frac{x}{l}-\frac{f n \beta}{k l}\right) \cos \left(\alpha x+\varphi_{n}+\beta n x-f n^{2}{ }_{\beta} 2 / 2 k\right)
$$

where $l(x)$ has been written to show the orders separated. (For the insensitivity of eq. (3) to the $\gamma$ of the film, see ref. 6. Photographic films do not reproduce spatial frequencies which exceed some resolution limit. In applications to $X$-ray wavefront-reconstruction microscopy, $\beta$ will be many orders of magnitude greater than the resolution capability of the film. Consequently each term of the second sum of eq. (3), (except for $n=0$ ) would be averaged out during the recording process. For high resolutions, the transmittance $H(x)$ should however be given by an equation of the form

$$
\begin{aligned}
H(x) \propto 2 A_{0}^{2}+\gamma \sum_{n} \text { rect }\left(\frac{x}{l}-\frac{f n \beta}{k l}\right) A_{n}^{2}-A_{0} \gamma \sum_{n} \text { rect }\left(\frac{x}{l}-\frac{f n \beta}{k l}\right) \exp \left[\mathrm{i}\left(\alpha x+\varphi_{n}-f n^{2} \beta^{2} / 2 k\right)\right] \\
-A_{0} \gamma \sum_{n} \text { rect }\left(\frac{x}{l}-\frac{f n \beta}{k l}\right) \exp \left[-\mathrm{i}\left(\alpha x+\varphi_{n}-f n^{2} \beta^{2} / 2 k\right)\right]
\end{aligned}
$$

rather than simply by the equation which one would obtain by recording the intensity of eq. (3). The transmittance described by eq. (4) could be realized from the intensity given by eq. (3) for instance by first "rectifying" the third term in eq. (3) by means of an optical equivalent of a "half-wave rectifier", comparably to half-wave rectification of a modulated radio-frequency signal, when producing an audio signal in a loudspeaker [14]. Several means of realizing the recording of eq. (4) as well as additional details and background will form the subject of a subsequent paper.

A highly manified and well resolved image of the scattering object can be "reconstructed" using the "high-resolution hologram" of eq. (4) by means of an optical system, such as that shown in fig. 1.

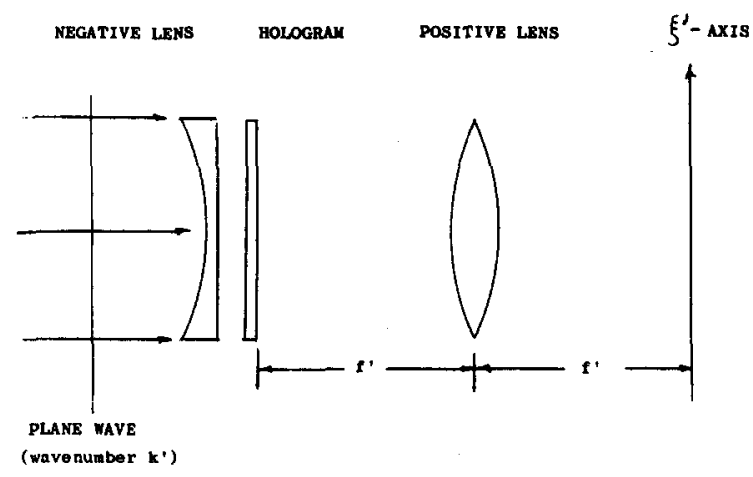

Fig. 1.

The plane wave incident on the hologram in fig. 1 is diffracted in the usual way into a central beam, representing the first two terms of eq. (4), an upper beam, representing the third term of eq. (4), and a lower beam, representing the fourth term of eq. (4). The negative lens serves to cancel the phase factor $f n^{2} \beta^{2} / 2 k$ in the third term of eq. (4), while the positive lens serves to effect the Fourier transform of the wavefront leaving the surface of the hologram. The final "image" is produced by the Fourier transformation of the third term of eq. (4), as carried out by the positive lens. If the wave-number of the light used in the reconstruction process (for instance laser light) is $k^{\prime}$, then the image produced by the third term will be proportional to 


$$
\begin{aligned}
\int \sum_{n} \operatorname{rect}\left(\frac{x}{l}-\frac{f n \beta}{k l}\right) A_{n} \exp \left[\mathrm{i}\left(\alpha x+\varphi_{n}-\frac{k^{\prime}}{f^{\prime}} \xi x\right)\right] \mathrm{d} x \cong 2 l \sum_{n} A_{n} \exp \left[\mathrm{i}\left\{\varphi_{n}+\left(\alpha-\frac{k^{\prime}}{f^{\prime}} \xi\right) \frac{f n \beta}{k}\right)\right]= \\
=2 l \sum_{n} A_{n} \exp \left[\mathrm{i}\left(\varphi_{n}+n \frac{\beta}{M} \xi^{\prime}\right)\right]
\end{aligned}
$$

where $\xi^{\prime}=\xi+\alpha f^{\prime} / k \quad$ and

$$
M=k f^{\prime} / k^{\dagger} f
$$

is the magnification characteristic of the overall two-wavelength process. $(f=$ distance of the scattering object from the hologram in the construction process, and $f^{\prime}=$ focal length of the positive lens used in the reconstruction process). .It will be noted that the series in eq. (5) has indeed the same form as the series representing the scattering object described by eq. (1), and one may therefore conclude that a well-resolved, magnified "image" of the scattering object has been reproduced along the $\xi$ ' axis, without losing the general simplicity and speed which are characteristic of holography [1-6].
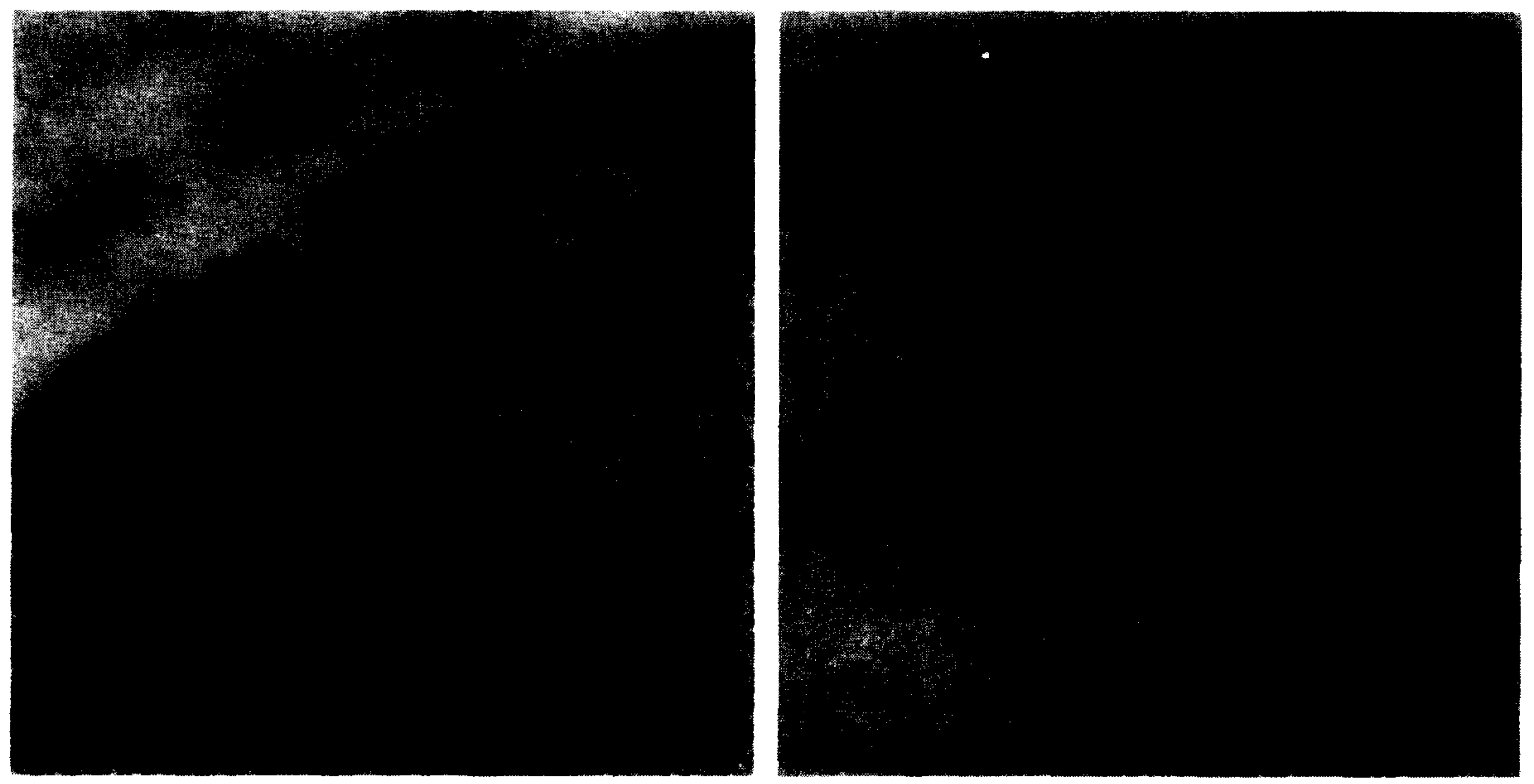

Fig. 2.

A greatly magnified $(\mathbf{1 5 0} \times)$, well resolved micro-photograph of a portion of a fly's wing (fig. $2 a$ ) was reconstructed from a hologram such as that shown in fig. $2 b[5,6]$ by illuminating the hologram in light diverging from a point source, and by placing the film at a distance $f^{\prime}$ from the hologram. The entire recording and reproduction process was carried out in $6328 \AA$ laser light without the aid of any focussing elements. Fig. 2 serves to illustrate the focussing (lens-like) and magnifying properties known to be inherent in holograms [1,2] (see also e.g. ref. 6), and more particularly here the $f^{\prime} / f$ factor in eq. (6).

It is a pleasure to acknowledge here the significant contributions to this work by John Winthrop, as well as the constant inspiration received from and the many fruitful discussions held with Professors S. Krimm, L. J. Oncley, C. R. Worthington and Dr.H. M. A. El-Sum. We also wish to thank D. Brumm, as well as E. N. Leith, J. Upatnieks and A. Funkhouser for assistance in obtaining the photographs of fig. 2. Finally it is a pleasure for one of us (GWS) to acknowledge very fruitful conversations with Professors D. Gabol and P. Kirkpatrick, as well as with Dr. A. Lohmann, and to thank Dr. J. Blaise and Prof. Roizen-Dossier for assistance in locating the reference to the rect notation used in the equations. 
1. D. Gabor, Nature 161 (1948) 777.

2. D. Gabor, Proc. Roy Soc. (London) A197 (1949) 454.

3. P. Kirkpatrick and H.M.A.E1 Sum, J. Opt. Soc. Am. 46 (1956) 825.

4. A. Lohmann, Optica Acta, 3 (1956) 97.

5. E.N. Leith and J. Upatnieks, J. Opt. Soc. Am. 54 (1964) 1295.

6. G.W.Stroke and D.G.Falconer, Theoretical and experimental foundations of wavefront-reconstruction imaging, in Symposium on Optical and electro-optical information processing technology, ed, J.T.Tippett, L.C.Clapp, D. Berkowitz and C.J.Koester (M.I.T.Press, 1964) in print.

7. M. J. Buerger, J. Appl. Physics 21 (1950) 909.

8. J.C. Kemdrew, G. Bodo, H.M.Dinitz, R.G.Parrish, H.Wyckoff and D.C.Phillips, Nature 181 (1958) 662.

9. J.C. Kendrew, Three-dimensional structure of globular proteins, in Biophysical Science - A study program, ed: J.L. Oncley (John Wiley and Sons, New York, 1959) p. 94.

10. M. Born and E. Wolf, Principles of optics, 2nd revised edition, (Pergamon press, New York, 1964 ), p. 453.

11. E.g. P.M. Woodward, Probability and information theory, with applications to radar, (2nd impression, 1960), (Pergamon Press London, 1953) p. 28.

12. G.W.Stroke, An introduction to optics of coherent and non-coherent electromagnetic radiations (The University of Michigan, Engineering Summer Conferences on Lasers, May 1964), 77 pages.

13. G.W.Stroke, Diffraction gratings, in Handbuch der Physik, Vol. 29, ed: S. Flftgge (Springer Verlag, Berlin and Heidelberg), in print.

14. S.Seely, Electron-tube circuits, (McGraw Hill Book Company, New York, 1958) p. 194.

$$
\text { ***** }
$$

\title{
POLARISATION (NEAR THRESHOLD) OF THE SPECTRAL LINES $\lambda=5791 / 90 \AA$ AND $\lambda=5770 \AA$ OF MERCURY, EXCITED BY ELECTRON IMPACT
}

\author{
H. G. M. HEIDEMAN \\ Fysisch Laboratorium der Rijksuniversiteit Utrecht, Nederland
}

Received 23 November 1964

Earlier research by Skinner and Appleyard $[1,2]$ has shown that radiation emitted by mercury vapour, when excited by a beam of electrons, is polarised. They observed that in most cases the degree of polarisation as a function of the energy of the bombarding electrons approaches zero at threshold. This, however, is contradictory to theory [3-5]. For helium, experiments [6] have suggested that the polarisation does not go to zero at threshold.

By using improved methods of measurement (smaller energy spread in the electron beam and a more sensitive way of measuring small light intensities) we succeeded in measuring the polarisation close to threshold more accurately.

Measuring method. A short description of the apparatus follows here; for a more detailed one see $[7,8]$. The sealed excitation tube contains mercury vapour, that can be excited by means of a beam of electrons of adjustable velocity. The mercury vapour pressure is the saturated vapour pressure at a given temperature of the tube wall. A narrow cross section of the elec- tron beam is imaged onto the entrance slit of the monochromator in such a way that the light enters from a direction at right angles to the electron beam. The light of a selected spectral line coming from the monochromator passes through a polaroid filter and falls on the photo-cathode of a multiplier tube. Each photo-electron gives a current pulse at the output of the multiplier. These pulses are amplified with a fast amplifier (rise time $0.5 \mu \mathrm{sec}$ ) and finally recorded with a counting device.

The percentage degree of polarisation $P$ is defined as follows

$$
P=\left(I_{\|}-I_{\perp}\right) /\left(I_{\|}+I_{\perp}\right) \times 100 .
$$

By rotating the polaroid filter we measure successively the intensity of the light with the electrical vector parallel and perpendicular to the beam, here denoted by $I \|$ and $I_{\perp}$, respectively. In our case the polaroid filter is rotated at 25 $\mathrm{r} / \mathrm{sec}$. The pulses from the photo-multiplier are supplied to a two-channel electronic switch, which operates synchronically with the rotating 